\title{
ALÍVIO DA DOR CRÔNICA NÃO NEOPLÁSICA COM OPIÁCEOS
}

\author{
Cibele Andrucioli de Mattos Pimenta* \\ Manoel Jacobsen Teixeira** \\ Cláudio Fernandes Correa*** \\ Fabiana S. Müller**** \\ Fabricia C.G.Goes***** \\ Raphael M. Marcon******
}

PIMENTA, C.A.de M.; TEIXEIRA, M.J.; CORREA, C.F.; MÜLLER, F.S.; GOES, F.C.G.; MARCON, R.M. Alívio da dor crônica não neoplásica com opiáceos. Rev.latino-am.enfermagem, Ribeirão Preto, v. 7, n. 4, p. 65-73, outubro 1999.

O uso de opiáceos para o controle da dor crônica não relacionada ao câncer é controverso. Avaliar o alívio da dor e as mudanças nas atividades de vida diária advindas da infusão intratecal de opiáceos em 11 doentes, com dor crônica não neoplásica, foram os objetivos deste estudo. Todos os doentes haviam previamente sido submetidos a diversas modalidades terapêuticas sem alivio significativo. Os doentes foram solicitados a avaliar as características da dor e as atividades de vida diária comparandoas antes e após o tratamento com opiáceo intratecal por meio de sistemas implantados. Observou-se alívio da dor. No entanto, melhora da funcionalidade não foi observada com a mesma magnitude.

UNITERMOS: dor, dor crônica, dor não oncológica, opiáceo, alivio da dor, qualidade de vida, dor neuropática, dor miofascial

\section{INTRODUÇÃO}

Os princípios gerais relacionados ao uso de analgésicos opiáceos para o alívio da dor aguda, de moderada a intensa, e da dor crônica, relacionada ao câncer, estão bem estabelecidos na literatura ${ }^{1,6,9}$. Há controvérsias quanto ao uso de opiáceos para o controle da dor crônica não oncológica. As controvérsias referemse à eficácia destes fármacos para o controle da dor neuropática e músculo-esquelética, à ocorrência de toxicidade orgânica, à magnitude dos efeitos colaterais, ao desenvolvimento de dependência física e tolerância e, principalmente, quanto à possibilidade do desenvolvimento de dependência psíquica $^{7,10,11,12,14,17,21,22}$. Há conceito muito difundido de que o uso de opiáceos para o controle da dor crônica é inapropriado ${ }^{4,5,7,14}$ mas muitos especialistas julgam que parcela de doentes com dor crônica poder-se-ia beneficiar com a utilização destes analgésicos ${ }^{8,10,11,14,16,17,20,21,22}$. Nos últimos anos diversos conceitos foram e estão sendo revistos e busca-se definir critérios para a indicação de opiáceos e identificar quais doentes seriam mais susceptíveis a apresentarem resultados piores ${ }^{10,12,13,14,15}$.

Os opiáceos produzem analgesia ligando-se a receptores de encefalinas ou endorfinas no encéfalo, medula espinhal e sistema nervoso periférico. Atuam modulando a atividade nociceptiva através de numerosos mecanismos ${ }^{3,18}$. Compreendem um grupo heterogêneo de drogas naturais, sintéticas e endógenas (encefalinas, endorfinas e dinorfinas) com propriedades químicas semelhantes à da morfina. São classificados como agonistas (morfina, codeína, heroína, oxicodone,

\footnotetext{
* Enfermeira. Professora Doutora do Departamento de Enfermagem Médico-Cirúrgica da Escola de Enfermagem da Universidade de São Paulo. Coordenadora de Enfermagem da Liga de Dor do Hospital das Clínicas da Faculdade de Medicina da Universidade de São Paulo

** Neurocirurgião. Professor Doutor do Departamento de Neurologia da Faculdade de Medicina da Universidade de São Paulo. Chefe do Ambulatório de Dor da Divisão de Clínica Neurológica do Hospital das Clínicas da Faculdade de Medicina da Universidade de São Paulo

*** Neurocirurgião. Médico do Ambulatório de Dor da Divisão de Clínica Neurológica do Hospital das Clínicas da Faculdade de Medicina da Universidade de São Paulo

**** Enfermeira. Ex-integrante da Liga de Dor do Hospital das Clínicas da Faculdade de Medicina da Universidade de São Paulo ***** Enfermeira. Ex-integrante da Liga de Dor do Hospital das Clínicas da Faculdade de Medicina da Universidade de São Paulo

****** Aluno de graduação da Faculdade de Medicina da Universidade de São Paulo
} 
metadona, propoxifeno, meperidina, fentanila), agonistas parciais (buprenorfina), agonista/antagonista (pentazocina, nalbufina) e antagonista (naloxona), de acordo com sua capacidade de sensibilizar os receptores opiáceos. Há relatos de complicações decorrentes de uso inadequado, intoxicação aguda e hiperdosagem com todas estas drogas. A administração repetida destes fármacos possibilita o aparecimento de efeitos de tolerância, dependência física e dependência psíquica, em graus variados ${ }^{3}$. Tolerância é a necessidade de aumento de dose para a obtenção do mesmo efeito analgésico. Dependência física é um fenômeno caracterizado pelo aparecimento de síndrome de abstinência quando a droga é descontinuada de modo abrupto ou quando um antagonista é administrado. A diminuição gradativa da droga previne o aparecimento de síndrome de abstinência ${ }^{3,9}$. Tolerância e dependência física são efeitos farmacologicamente previsíveis, advindos da ação competitiva dessas drogas sobre os neuropeptídios naturalmente produzidos pelos indivíduos. Estes eventos parecem não constituir limitações para o uso terapêutico ${ }^{3,14}$. Dependência psicológica ou vício é definido como o uso compulsivo de substâncias psicoativas apesar do prejuízo físico, emocional e social advindos. Há perda de controle, uso compulsivo, continuado e envolvimento global na procura e uso da $\operatorname{droga}^{3,14}$. Sabe-se que o desenvolvimento de dependência psicológica pode ser independente do desenvolvimento de tolerância e dependência física, embora os fenômenos sejam estreitamente relacionados ${ }^{3}$. Não se conhece a real ocorrência de dependência psicológica entre doentes com dor crônica que utilizam opiáceos. Este desconhecimento está, possivelmente, relacionado à falta de consenso e critérios que definam dependência psicológica e da confusão entre os conceitos de tolerância, dependência física e dependência psíquica ${ }^{7,9,10,11,12}$.

A preocupação com a seleção de critérios de indicação e com a identificação de grupos de doentes mais e menos susceptíveis ao efeito analgésico tem mobilizado os estudiosos do tema. De acordo com SCHOFFERMAN $^{14}$ os opiáceos devem ser indicados para doentes com doença estruturada, onde a queixa de dor e incapacidade são coerentes com a história e quadro físico, os aspectos emocionais e sociais são compatíveis ao quadro álgico e o doente não deve possuir antecedentes de uso de álcool ou drogas. Segundo o autor, estes doentes são os que mais se beneficiam com essa terapêutica mas, infelizmente, constituem minoria entre os doentes com dor crônica não neoplásica. Opiáceos não devem ser indicados para doentes com estilo de vida centrado na dor, pois a dor e o opiáceo passarão a ser o núcleo de atenção do indivíduo. Neste grupo de doentes freqüentemente observa-se que o relato de dor e incapacidade são desproporcionais e que fatores psicológicos e sociais preponderam no quadro clínico. Este grupo, embora seja o mais numeroso entre os doentes com dor crônica, parece ser o que menos se beneficia com o opiáceo ${ }^{14}$.

TENNANT \& UELMEN $^{20}$; PORTENOY ${ }^{10} \mathrm{e}$ SCHUG \& LARGE ${ }^{15}$ sugerem critérios para a admissão, para a condução da fase de teste e normas para a manutenção de doentes com dor crônica em protocolos com opiáceos. As diretrizes para a indicação são: falência de resultados com métodos analgésicos tradicionais; avaliação física e emocional preferencialmente por 2 especialistas; ausência de história anterior de uso de drogas e álcool, embora a existência de antecedentes constitua contra-indicação relativa, requeira consulta com especialistas em droga-adição e consulta ao serviço de controle de drogas; a decisão sobre a admissão de doentes no protocolo deve ser tomada pela equipe de profissionais ( 2 ou mais); obter-se, por escrito, o consentimento do doente e dar-se ênfase na melhora da atividade física e social decorrente do alívio da dor. Propõem como esquema básico fase de teste com duração de 4 semanas e o uso de opiáceo agonista "mu" de longa duração; o uso da via oral em regime de horário pré-establecido e avaliações freqüentes para rever a dosagem, avaliar a eficácia analgésica e identificar alívio duradouro da dor e/ou melhora da função. Propõem que o uso do opiáceo em regime prolongado deve atentar para os seguintes aspectos: prescrição por 1 único profissional; revisões mensais do alívio, do estado funcional, do uso apropriado da medicação e do aparecimento de efeitos colaterais; revisão e discussão contínua dos aspectos educativos relativos à dor crônica e seu manejo; continuidade dos esforços para se obter melhora física e social como resultado do alívio da dor; contrato com o doente explicitando o uso apropriado do fármaco e a possibilidade de interrupção do tratamento e notificação ao serviço de controle de entorpecentes nas situações de uso recreacional, perdas ou escalada de dose injustificáveis e solicitação de opiáceos em outros serviços; uso de supervisores ou internação para avaliação no caso de exacerbação inexplicável da dor ou aumento injustificável da dose e, finalmente, a reavaliação continuada da situação em sua globalidade considerando outros métodos para o controle da dor.

A dor neuropática e por afecções do aparelho locomotor é difícil de ser tratada. Foi observada melhora da dor crônica neuropática e músculo-esquelética resultante do uso dos opiáceos por alguns autores ${ }^{8,11,16,22}$, mas poucos ${ }^{20,22}$ relatam também melhora da funcionalidade. Há quem julgue que para a maioria dos doentes o uso do opiáceo compromete de modo significativo a reabilitação ${ }^{4,5,7,14}$ e realçam que a melhora do sintoma sem melhora da função não é suficiente para a continuidade da terapia. 
Estudo que envolveu 1912 médicos norte americanos, de diferentes especialidades, avaliou as crenças destes profissionais quanto ao uso de opiáceos para o controle da dor crônica não relacionada ao câncer. Solicitou que estes atribuíssem notas a algumas assertivas. Observou-se que os médicos prescrevem opiáceos para o controle da dor crônica não oncológica de modo não freqüente (média - 1,42); que preocupam-se em avaliar a melhora da funcionalidade do doente que utiliza estes fármacos (média - 4,41) e que o alívio da dor sem melhora da funcionalidade é justificativa para continuar o uso de opióides para significativa parcela dos entrevistados (média- 2,51) ${ }^{21}$.

Estudos sobre este tema são ainda pouco freqüentes em países desenvolvidos e desconhece-se a existência de avaliações sistemáticas em nosso meio. $\mathrm{O}$ objetivo deste trabalho foi verificar o alívio da dor e as mudanças nas atividades de vida diária antes e após a infusão dos agentes morfínicos no sistema nervoso central, através de sistemas implantados, em doentes com dor crônica não oncológica. Visou também caracterizar a ocorrência de efeitos colaterais, a progressão da dose do analgésico e identificar os receios dos doentes quanto à terapia utilizada.

\section{METODOLOGIA}

\subsection{Local, período e casuística}

O estudo foi desenvolvido no Ambulatório de Dor da Clínica Neurológica do Hospital das Clínicas da Faculdade de Medicina da Universidade de São Paulo. Os dados foram coletados no período entre outubro e dezembro de 1995. Compuseram a amostra doentes com dor crônica não relacionada ao câncer que, naquele momento, recebiam opiáceos por via intratecal, atendidos no Ambulatório de Dor e que concordaram em participar da pesquisa, com perfazendo 11 indivíduos adultos, com idade variando entre 32 e 72 anos (média de 50,8 anos) sendo 6 mulheres e 5 homens. A dor era de origem neuropática em 5 doentes e miofascial em 6 .

\subsection{Métodos}

Utilizando-se instrumento construído especialmente para o estudo, os doentes foram entrevistados uma única vez e solicitados a avaliar a dor e as atividades de vida diária comparando-as antes e após o tratamento com opiáceo intratecal. A intensidade da dor foi avaliada por meio de escala numérica de 0 a 10 . As atividades de vida diária como trabalho, atividade física, autocuidado, recreação, sono, atividade sexual e as alterações de humor e na capacidade de concentração foram investigadas por meio de questões fechadas, contendo 5 graduações, onde se indagava se o tratamento em questão não interferiu, melhorou, melhorou muito, piorou ou piorou muito a capacidade/habilidade do indivíduo para o desempenho das atividades acima especificadas. As preocupações, receios e conceitos do doente quanto ao método terapêutico utilizado foram investigados por meio de questões abertas. Os dados relativos à concentração e dose do remédio, tempo de tratamento, tratamentos anteriores e diagnóstico da dor foram levantados do prontuário e do relato dos doentes. Todos os doentes consentiram em participar do estudo.

\section{RESULTADOS}

Os doentes submeteram-se a tratamentos anteriores com fármacos por via oral tais como: antiinflamatórios não hormonais (10), opiáceos (6), antidepressivos (5), neurolépticos (5) e anticonvulsivantes (5) durante 3,9 anos, em média. O resultado foi avaliado pelos doentes como regular ou ruim em $82 \%$ das vezes. Dos 11 doentes avaliados 9 informaram terem se submetido a tratamentos como: alongamento (5), acupuntura (4), infiltração com lidocaína (4), calor (3), hidroterapia (3), TENS******* (2), corrente farádica (1), massagem (1) e ultra-som (1). Os resultados foram referidos como regular ou ruim em $86 \%$ dos casos. Os doentes relataram terem sofrido diversos procedimentos cirúrgicos como: descompressão de cauda equina (5), rizotomia de faceta (4), remoção de tumor espinhal (4), exérese de prolapso de disco intervertebral (3), descompressão do cordão medular (2), estimulação medular (2) e descompressão de nervo periférico (1). Submeteram-se, em média, à 1,9 procedimentos cirúrgicos. Os resultados foram avaliados como moderados em $20 \%$ das vezes e ruins em $80 \%$ dos casos.

A duração da queixa álgica variou entre 2 e 15 anos e a média foi de 5,3 anos. Dos avaliados 7 informaram ter dor entre 2 e 5 anos e 4 entre 6 e 15 anos.

O tempo de uso do opiáceo intratecal foi em média de 19,6 meses, variando entre 2 e 60 meses. O opiáceo utilizado por 10 doentes foi o cloridrato de morfina e 1 doente utilizou tramadol. A dose diária inicial média da morfina foi $2 \mathrm{mg}$, variando entre $1 \mathrm{mg}$ e $4 \mathrm{mg}$. A dose diária média do cloridrato de morfina no momento da avaliação para este estudo foi de 1,55 mg variando

******* TENS - O método de estimulação elétrica transcutânea consiste de sistema com gerador de impulsos elétricos e eletrodos. Estímulos elétricos não dolorosos, que podem ser controlados em freqüência e intensidade, são aplicados sobre a região da dor 
entre $0,5 \mathrm{mg}$ e $3 \mathrm{mg}$. O doente que recebia tramadol iniciou o tratamento com $60 \mathrm{mg}$ diárias e no momento da avaliação utilizava a mesma dose. A dose do opiáceo permaneceu estável em 4 doentes, diminuiu em 4 e aumentou em 3 doentes. Alguns doentes relataram períodos em que ocorreu flutuação da dose, decorrente de piora do quadro álgico ou revisão cirúrgica do sistema de infusão.

Quanto à intensidade da dor antes do tratamento com opiáceo intratecal 9 doentes relataram dor intensa (entre 8 e 10), 1 referiu dor moderada (entre 4 e 7) e 1 doente não precisou a intensidade. A intensidade média observada foi de 8,6. Após o tratamento 4 doentes avaliaram sua dor como leve (entre 1 e 3), 6 disseram que a dor era moderada (entre 4 e 7 ) e 1 doente relatou dor intensa (entre 8 e 10). A média de intensidade da dor após o uso de opiáceo intratecal foi de 3,9, conforme pode-se observar na Figura 1.

A duração dos episódios diários de dor também foi avaliada pelos doentes. Antes da terapêutica com opiáceos 7 informaram sentir dor 6 ou mais horas por dia, 3 referiram que a duração da queixa álgica era entre 1 e 5 horas diárias e 1 doente disse que a duração dos episódios dolorosos era menor que 1 hora. Após o tratamento 1 doente disse não ter dor, 6 que a duração da dor era inferior à 1 hora diária, 1 doente referiu que a dor durava entre 3 e 5 horas diárias, 1 informou que a dor durava mais que 6 horas por dia e 2 doentes não precisaram a duração dos episódios dolorosos, conforme pode-se observar na Figura 2.

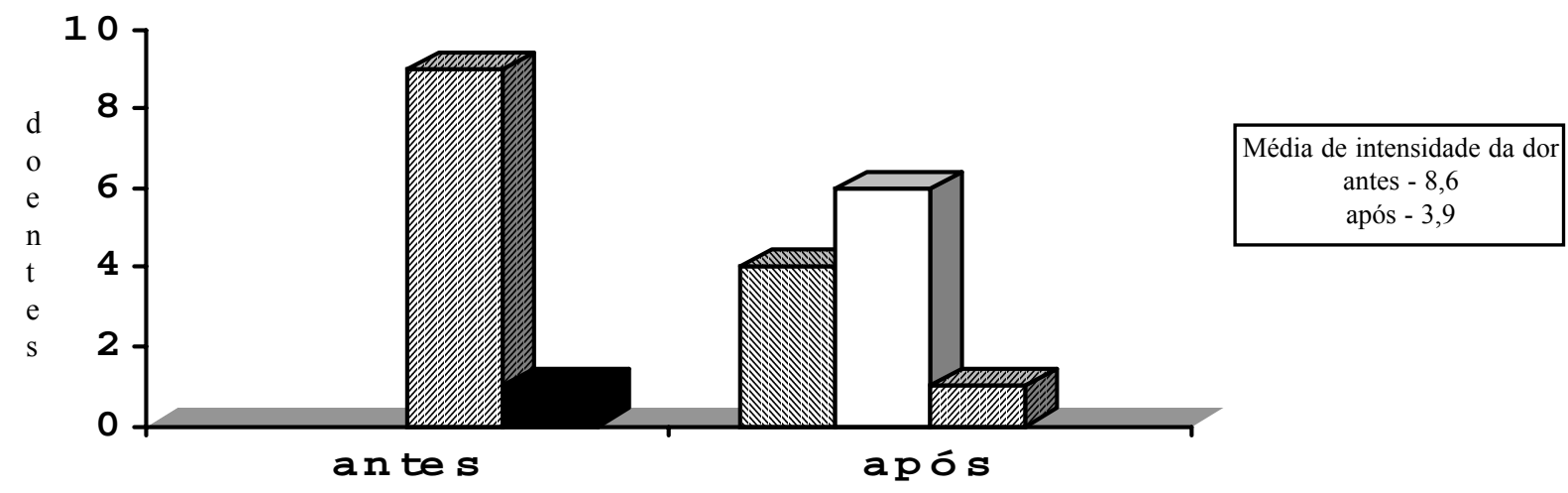

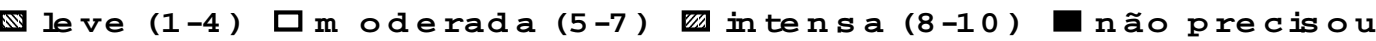

Figura 1 - Intensidade da dor antes e após a infusão de opiáceo. São Paulo, 1995



$\square$ sem dor $<1$

Wh $1 \mathbf{h}-5 \mathbf{h}$

$6 \mathrm{~h} \circ \mathrm{u}+$

não precis o u 
A avaliação do doente sobre sua capacidade para as atividades de vida diária, antes e após tratamento com opiáceo intratecal pode ser observada na Tabela 1.

Tabela 1 - Avaliação dos doentes quanto ao desempenho das atividades de vida diária, humor e capacidade de concentração, antes e após o tratamento com opiáceo intratecal. São Paulo, 1995

\begin{tabular}{|c|c|c|c|c|c|c|c|c|c|c|}
\hline \multirow{3}{*}{$\begin{array}{l}\text { ATIVIDADES } \\
\text { DE VIDA } \\
\text { DIARAA }\end{array}$} & \multirow{2}{*}{\multicolumn{2}{|c|}{ não se alterou }} & \multicolumn{4}{|c|}{ DOENTES } & \multicolumn{4}{|c|}{$(n=11)$} \\
\hline & & & \multicolumn{2}{|c|}{$\begin{array}{l}\text { melhorou ou } \\
\text { melhorou muito }\end{array}$} & \multicolumn{2}{|c|}{$\begin{array}{l}\text { piorou ou } \\
\text { piorou muito }\end{array}$} & \multicolumn{2}{|c|}{ não consta } & \multicolumn{2}{|c|}{ TOTAL } \\
\hline & $\mathrm{n}$ & $\%$ & $\mathrm{n}$ & $\%$ & $\mathrm{n}$ & $\%$ & $\mathrm{n}$ & $\%$ & $\mathrm{n}$ & $\%$ \\
\hline Trabalho & 5 & 5,68 & 3 & 3,41 & 2 & 2,27 & 1 & 1,14 & 11 & 12,50 \\
\hline Atividade fisica & 4 & 4,55 & 4 & 4,55 & 3 & 3,41 & 0 & 0 & 11 & 12,50 \\
\hline Autocuidado & 4 & 4,55 & 6 & 6,82 & 1 & 1,14 & 0 & 0 & 11 & 12,50 \\
\hline Lazer & 4 & 4,55 & 4 & 4,55 & 3 & 3,41 & 0 & 0 & 11 & 12,50 \\
\hline Hum or & 5 & 5,68 & 4 & 4,55 & 2 & 2,27 & 0 & 0 & 11 & 12,50 \\
\hline Concetração & 3 & 3,41 & 5 & 5,68 & 3 & 3,41 & 0 & 0 & 11 & 12,50 \\
\hline Atividade sexual & 6 & 6,82 & 1 & 1,14 & 1 & 1,14 & 3 & 3,41 & 11 & 12,50 \\
\hline Sono & 6 & 6,82 & 5 & 5,68 & 0 & 0 & 0 & 0 & 11 & 12,50 \\
\hline TOTAL & 37 & 42,05 & 32 & 36,36 & 15 & 17,05 & 4 & 4,55 & 88 & 100,00 \\
\hline
\end{tabular}

Pela Tabela 1 observa-se que dos 8 aspectos avaliados nos 11 doentes, em $42,05 \%$ das vezes os pacientes informaram que o tratamento com opiáceo não alterou sua condição prévia ou o desempenho destas atividades. Em 36,36\% do total de aspectos avaliados os doentes consideraram que o tratamento trouxe melhora e em 17,05\% dos casos foi referida piora da funcionalidade.

Os medos e receios expressados pelos doentes referem-se ao dispositivo bomba de infusão e à morfina. Dos avaliados 5 referiram ter medo de dano físico, 3 verbalizaram medo de ficar dependente da morfina, 2 disseram encarar a morfina como o ultimo recurso e 1 relatou ter medo de ficar com impotência sexual. Quando indagados sobre as vantagens e desvantagens do tratamento 9 doentes referiram como vantagem o alívio da dor e 2 disseram não perceber vantagem. Foram apontadas como desvantagens: alteração de auto-imagem e auto-estima (3), dependência médica (2), impotência (1), obstipação (1), síndrome de abstinência (1) e efeitos colaterais de forma geral (1). Dos 11 doentes avaliados 4 disseram não perceber desvantagens.

Quanto à existência de efeitos colaterais no início do tratamento 5 doentes referiram não os ter experienciado. Entre os 6 que relataram efeitos colaterais ocorreu: náusea/vômito (6), obstipação (3), prurido (2), retenção urinária (1) e síndrome de abstinência (1). A investigação da existência de efeitos colaterais na fase de coleta de dados para este estudo mostrou diminuição significativa: 7 doentes disseram não ter efeitos colaterais, 3 relataram sentir náuseas e 2 referiam obstipação. Finalmente, quando indagados sobre se recomendariam ou não este tratamento para alguém com problemas similares aos seus, 7 doentes disseram sim e 4 não. As justificativas foram alívio da dor (4), melhora da funcionalidade (1) e ausência de alternativas (2).

\section{DISCUSSÃO}

Os doentes deste estudo apresentavam dor crônica de origem neuropática e miofascial por período prolongado (variando entre 2 e 15 anos) e haviam se submetido a diversas terapias analgésicas farmacológicas, fisioterápicas, cirúrgicas ablativas e de neuroestimulação, sem resultado satisfatório. A opção pelo uso de opiáceo não foi, portanto, estratégia de início de tratamento, o que está de acordo com as recomendações de literatura e experiência de outros autores ${ }^{11,15,16,22}$.

O tempo de uso do opiáceo foi prolongado $(19,6$ meses em média) e a dose média no início do tratamento foi de $2 \mathrm{mg}$ e no final de $1,55 \mathrm{mg}$. Dos avaliados 4 mantiveram a mesma dose, em 4 houve necessidade de diminuição e em 3 foi necessário aumentar-se a dose. Nos doentes em que se aumentou a dose este aumento foi da ordem de 2 ou 3 vezes a dose inicial no prazo que variou entre 1 e 2,5 anos. A preocupação em se avaliar o aumento da dose visava a busca de sinais de tolerância e dependência psíquica.

A administração prolongada de opiáceos agonistas puros, agonistas parciais ou agonista/ antagonista acarreta desenvolvimento de tolerância, em graus variáveis. Tolerância aguda refere-se à situação em que o desenvolvimento de tolerância é muito rápido, após uma única ou poucas doses do fármaco. Tolerância crônica refere-se à situação em que a droga é administrada 
por tempo prolongado antes de ocorrer diminuição no efeito. É aceito que tolerância absoluta aos opiáceos não ocorre. Tolerância cruzada é aquela situação onde a tolerância a uma droga confere tolerância à outra. A tolerância aos diversos efeitos dos opiáceos desenvolvese em ritmos diferentes. De modo geral, a tolerância aos efeitos colaterais dos opiáceos desenvolve-se mais rapidamente que a tolerância ao efeito analgésico e o desenvolvimento de tolerância à depressão respiratória é mais rápido que a tolerância à ação obstipante. Pode haver tolerância após alguns dias de infusão contínua de opiáceos para controle da dor aguda e contrariamente a isto pode-se observar doentes com doença oncológica estável recebendo doses estáveis de opiáceo por prolongados períodos de tempo. Se um doente com dor crônica não oncológica necessita continuamente de doses altas e crescentes de opiáceos para a analgesia deve-se considerar a possibilidade de interromper o tratamento ${ }^{3,12,13}$. As doses utilizadas pelos doentes deste estudo podem ser consideradas pequenas e não se observou escalação de dose.

A avaliação do desenvolvimento de dependência psíquica (vício) no doente com dor não é fácil. O conceito de vício pode incluir sintomas de dependência física e tolerância e estes, por si só, não significam vício. No doente com dor crônica que recebe opiáceo os fenômenos de tolerância e dependência física podem ser esperados. O comportamento inadequado associado à busca incessante da droga, a preocupação constante com a sua falta, a continuidade do uso apesar dos danos físicos, emocionais e sociais decorrentes, a perda de controle sobre a ingestão do fármaco e a ausência de efeito analgésico significativo, é o dado mais importante para o diagnóstico de vício ${ }^{17}$. Segundo SAVAGE ${ }^{12,13}$ os sinais sugestivos de vício na terapia com opiáceos para o controle da dor crônica são: não aceitação de outras alternativas terapêuticas propostas, relato de não alívio da dor com outro método que não o opiáceo, preferência por opiáceos de ação curta ou em bolo, obtenção de prescrição em múltiplos serviços, uso de drogas de rua, não adesão à outras recomendações terapêuticas, diminuição do nível funcional, apesar da aparente analgesia, persistência de efeitos colaterais como sonolência, sedação e euforia, não ingestão dos fármacos conforme prescritos, a medicação freqüentemente acaba antes do período previsto e relatos repetidos de perda ou extravio da prescrição. Um único sinal não necessariamente indica vício, mas uma constelação de sinais pode ser sugestivo. Nos doentes deste estudo não foram observados comportamentos como os acima descritos. SAVAGE ${ }^{12,13}$ considera, ainda, que os critérios para desordens relacionadas ao uso de substâncias proposto pela Diagnostic and Statistical Manual of Mental Disorders, DSM- III R, não são adequados para o diagnóstico de adição no doente com dor crônica pois 5 dos 9 critérios preconizados referem-se à dependência física e tolerância, que são freqüentes em doentes que utilizam opiáceos por tempo prolongado. Apenas os 4 critérios que se referem funcionalidade podem ser indicativos de adição nestes doentes (ingestão diferente do modo prescrito, repetidos relato de perda e solicitação de medicação em centros diferentes, uso continuado da medicação, apesar dos efeitos colaterais e piora do nível funcional, apesar da aparente analgesia).

Acredita-se que o efeito euforizante dos opiáceos seria o que levaria à adição. Há evidências que os opiáceos aumentam a atividade dos neurônios dopaminérgicos no córtex frontal, tegmental ventral e nucleo accubens, por inibir a recaptação de dopamina e, deste modo produz euforia. Embora este efeito ocorra, no uso clínico de analgésicos opiáceos tem-se observado que os doentes referem mais disforia do que euforia ${ }^{3,17}$. O potencial para adição não reside somente nas propriedades dos opiáceos, mas requer interação entre estas propriedades e variáveis fisiológicas, psicológicas e sociais e, a resultante desta interação, poderá predispor o indivíduo à dependência psíquica ${ }^{10}$.

O termo "pseudo-vício" refere-se à percepção do profissional de comportamentos semelhantes aos apresentados por aqueles com dependência psíquica mas que, de fato, estão associados à busca de analgesia em doentes com dor intensa, medicados de modo insuficiente e que não receberam tratamento efetivo para o controle da dor. O doente com "pseudo-vício" pode ser diferenciado daquele com dependência psíquica observando-se que quando doses mais elevadas do opiáceo são oferecidas o doente não as utiliza de modo a ter sedação ou euforia persistentes, a capacidade funcional melhora e a medicação é utilizada como prescrita, sem perda de controle ${ }^{12,13}$.

Intrigante é a especulação de que o uso prolongado de opiáceos pode induzir estado de hiperexcitabilidade do sistema nervoso central, semelhante à dor patológica ${ }^{15}$.

A média da intensidade da dor referida pelos doentes após o tratamento com opiáceo intratecal $(3,9)$ foi significativamente inferior à media da intensidade da dor antes do tratamento $(8,7)$. Também a duração diária dos episódios dolorosos apresentou mudança substancial: antes da terapia 7 doentes informaram sentir dor 6 ou mais horas por dia e após o tratamento 1 doente referiu dor 6 ou mais horas por dia. Antes da terapia 1 doente informou episódios dolorosos diários com menos de 1 hora de duração e após a infusão de opiáceo intratecal este dado foi relatado por 6 doentes.

A responsividade da dor neuropática aos opiáceos é questionável. Há controvérsias se a não responsividade é absoluta, como julgam alguns, ou se 
algum grau de alívio pode ser obtido em parcela de doentes, como creêm outros. Parece não se tratar de fenômeno do tipo "tudo" ou "nada". A avaliação da resposta da dor aos opiáceos pode ser feita mediante a administração e aumento da dose do fármaco até que os efeitos colaterais sejam significativos, sem analgesia adequada.

Outros autores também observaram melhora da magnitude da dor com o uso de opiáceos para controle da dor neuropática ou músculo-esqueletal ${ }^{8,11,16,22}$. PORTENOY \& FOLEY ${ }^{11}$ avaliaram retrospectivamente 38 doentes com dor crônica não oncológica que utilizaram opiáceo por via oral por, no mínimo 6 meses. Os fármacos mais utilizados foram oxicodona, metadona e levofarnal. A maioria dos doentes (24) referiu alívio da dor aceitável ou total e 14 relataram alívio inadequado. Observaram poucos ganhos no trabalho e na atividade social e em 2 doentes $(5,2 \%)$, com história de uso anterior de droga, o manejo do opiáceo tornou-se problemático. SHULZECK et al. ${ }^{16}$ avaliaram a ação analgésica da morfina por via oral em 60 doentes com dores neuropática e músculoesquelética não relacionadas ao câncer. Observaram que 23 doentes não relataram alívio, e em 14 doentes os efeitos colaterais foram tão intensos que houve necessidade de se interromper a terapia. Dos 23 doentes restantes 10 relataram alívio duradouro e efeitos colaterais toleráveis. Observou ainda que $43 \%$ dos doentes precisaram de aumento de dose e que a dosagem de opiáceo na urina em 45 doentes mostrou que 18 deles haviam interrompido a ingestão. ZENS et al. ${ }^{22}$ estudaram 100 doentes com dor neuropática ou lombar. A avaliação foi feita em 2 momentos: 14 dias e 1 ano após o início da terapia. Os doentes receberam por via oral codeína, buprenorfina e morfina de ação lenta. Encontraram que 21 doentes não obtiveram alívio, 28 informaram alívio parcial e 51 referiram bom alívio da dor. Os efeitos colaterais observados após 1 ano de terapia foram: constipação $(45 \%)$, náusea ( $25 \%)$, tontura ( $30 \%)$, fadiga ( $5 \%$ ) e outros $(15 \%)$. MOULIN et al. ${ }^{8}$ testaram a hipótese de que a morfina por via oral alivia a dor crônica oriunda de tecidos moles ou músculo-esquelética que não responderam a terapias analgésicas anteriores. Os doentes do grupo experimental receberam morfina de liberação lenta $(60$ $\mathrm{mg}, 2 \mathrm{x}$ ao dia) e o grupo controle recebeu placebo ativo (benztropine). A intensidade e o alívio da dor foram avaliados semanalmente ( 9 semanas) e medidas psíquicas, o estado funcional e aspectos cognitivos foram avaliados no início e fim do estudo. Os doentes que receberam morfina relataram dor de menor intensidade que os do grupo controle e os efeitos colaterais foram mais freqüentes. Não se observaram outras diferenças.

Dos doentes avaliados neste estudo 5 disseram não ter tido efeitos colaterais e 6 relataram náusea/vômito (6), obstipação (3), prurido (2), retenção urinária (1) e síndrome de abstinência (1), na fase inicial da terapia. Os efeitos colaterais relatados no momento da avaliação foram: náusea (3), obstipação (2) e 7 doentes disseram não ter efeitos colaterais. Observa-se que os efeitos colaterais diminuíram com o tempo, o que pode ocorrer quando se utilizam opiáceos, pelo fenômeno de tolerância. Parece que os efeitos colaterais relatados pelos doentes deste estudo foram de menor magnitude do que os observados nos estudos de ZENS et al. ${ }^{22}$ e SHULZECK et al. ${ }^{16}$. Talvez isto se deva ao fato de que menores doses do fármaco são necessárias para a analgesia quando se utiliza a via intratecal. A doente que referiu síndrome de abstinência teve o sistema de infusão mecânico trocado por um sistema a gás, que permite infusão contínua, o que possibilitou o desaparecimento do fenômeno.

A auto avaliação dos 11 doentes sobre o seu desempenho em 6 atividades de vida diária (trabalho, atividade física, autocuidado, lazer, atividade sexual e sono) e relativo ao humor e capacidade de concentração, antes e após a infusão do opiáceo intratecal mostrou que, em $37(42 \%)$ das 88 observações feitas os doentes referiram que o quadro não se alterou. Em 32 (36,3\%) das observações os doentes disseram que sua habilidade ou condição para os aspectos avaliados havia melhorado ou melhorado muito e em 15 observações $(17 \%)$ os doentes disseram ter piorado ou piorado muito sua condição nos aspectos avaliados. Os itens que os doentes mais referiram melhora após o tratamento foram: autocuidado (6), capacidade de concentração (5) e sono (5). Se a melhora do quadro álgico foi significativa e a ocorrência de efeitos colaterais não foi tão importante, por que não se observou melhora da funcionalidade na mesma proporção? Há hipóteses para esta questão. A importância de se avaliar a qualidade de vida como critério de eficácia do tratamento reside no fato de permitir ao profissional retirar o foco da atenção da doença/dor para centrá-lo no indivíduo. O alívio da dor parece favorecer a melhora da qualidade de vida em parcela dos doentes. No entanto, nem sempre pode-se esperar correlação direta entre alívio da dor e melhora da qualidade de vida. Componentes biológicos, emocionais e sociais podem estar tão comprometidos, devido à prolongada duração da condição álgica ou a outras causas que, apenas o alívio da dor, não é suficiente para normalizá-los. Talvez o alívio da dor seja condição necessária, mas não suficiente para a melhora da qualidade de vida. É possível, ainda, que o método utilizado para verificar o desempenho nas atividades cotidianas não apresente sensibilidade adequada para identificar mudanças sutis. Alívio da dor sem melhora da funcionalidade foi observado em outros estudos ${ }^{8,11}$.

Alguns autores referem que o uso de opiáceo na dor crônica não oncológica piora a funcionalidade e dificulta a reabilitação em parcela dos doentes pois, entre 
outros, retira do indivíduo e deposita no remédio o controle da situação ${ }^{4,14,15,17,19}$. Consideram que os programas de controle da dor crônica devem aumentar a autonomia e, se possível, diminuir a dor ${ }^{2,14,15,19}$.

O conceito de eficácia pode ser influenciado pelo interesse do observador ${ }^{14}$. Parcela significativa dos doentes considera que a redução da dor é o melhor indicativo da eficácia do tratamento e que com o alívio da dor todos os outros problemas estarão resolvidos. Parte dos médicos também enfatiza o alívio da dor e outros julgam que como o alívio não é possível em algumas síndromes álgicas, a melhora da funcionalidade é o critério de eficácia. Para as empresas de seguro o melhor critério de eficácia é a redução dos custos. Para a maioria dos doentes deste estudo a eficácia da terapêutica foi avaliada pela redução da dor. Ao serem indagados sobre as vantagens do tratamento 9 referiram-se ao alívio da dor. Indagados se recomendariam este tratamento para outras pessoas com problemas similares aos seus, 7 disseram sim e 4 não. Os que recomendariam este tratamento o fariam pelo alívio da dor experienciado (4), pela melhora da funcionalidade (1) e pela ausência de alternativas (2). Os que não o recomendariam verbalizaram como justificativas: ausência de alívio da dor (2), distúrbio de auto-imagem e auto-estima (2) e devido aos efeitos colaterais (1). A dependência dos profissionais de saúde, pela necessidade de visitas periódicas para preencher o sistema de infusão, foi apontado por 3 doentes como desvantagem do método.

As preocupações que os doentes mencionaram ter quanto ao uso de morfina foram: medo de desenvolver dependência psicológica (3), ser o último recurso (2), impotência sexual (1) e 6 doentes disseram não ter nenhuma preocupação. Os doentes deste estudo conheciam o fármaco analgésico que utilizavam e os possíveis efeitos indesejáveis decorrentes de seu uso, o que é condição básica em qualquer tratamento e, em especial, nas terapias onde a existência de comportamentos que inflijam o contrato terapêtico estabelecido entre o doente e os profissionais indica a interrupção deste.

\section{CONCLUSÃO}

O uso de opiáceo para o controle da dor crônica não neoplásica é tema polêmico em todo o mundo e não constitui terapia de início de tratamento. Não se conhece quais e nem que parcela de doentes poder-se-ia beneficiar com o seu uso. Discute-se a real eficácia destes fármacos para o alívio da dor neuropática e muscular, questiona-se a melhora da qualidade de vida que resulta desta terapia, indaga-se qual a magnitude dos efeitos colaterais que acometem os doentes e qual o risco de desenvolvimento de dependência psicológica. A análise do uso de opiáceo intratecal para o alívio da dor e melhora da funcionalidade realizada em 11 doentes com dor neuropática e miofascial, desenvolvida a partir da auto-avaliação do doente, mostrou importante alívio da dor, nos aspectos de intensidade e duração dos episódios dolorosos diários. No entanto, a melhora do desempenho das atividades de vida diária não foi da mesma magnitude. Dos avaliados 4 mantiveram a dose do opiáceo intratecal, em 4 doentes houve necessidade de diminuí-la e em 3 houve aumento. Os efeitos colaterais não foram expressivos na maioria dos avaliados e não se observou comportamento de adição nestes doentes. Programas complementares visando a readaptação social, laborativa, emocional e física talvez possam contribuir para a melhora da qualidade de vida dos indivíduos.

Considera-se que este estudo preliminar subsidiará a organização de outras pesquisas relativas ao uso de opiáceos na dor crônica não neoplásica, que superem as limitações advindas de estudos retrospectivos, com pequena amostra, sem grupo controle e sem avaliação da estrutura e dinâmica psíquica e cognitiva antes, durante e após a terapia.

\section{NON ONCOLOGIC CHRONIC PAIN RELIEF WITH OPIOIDS}

Opioids for non cancer pain control are controversial. The evaluation of the pain relief, changes in quality of life and complications due to long term infusion of opioids in the lumbar subarachnoid space in 11 patients with non cancer pain were the goals of this study. Patients were previously treated with drugs and surgical procedures, without significant pain relief. Patients were asked to compare pain characteristics and daily life activities before and after this treatment. The long term spinal opioids through implantable pumps for non-oncologic pain produced pain relief but did not improve the quality of life in the majority of the cases.

KEY WORDS: pain, chronic pain, non malignant pain, opioids, pain relief, quality of life, neurophatic pain, miofascial pain

\section{ALIVIO DEL DOLOR CRONICO NO NEOPLÁSICO CON OPIÁCEOS}


dolor y los cambios en las actividades de la vida diaria debidas a la infusión intratecal de opiáceos, en 11 pacientes con dolor crónico no neoplásico fue el objetivo de este estudio. Todos los pacientes habían previamente sido sometidos a las diversas modalidades terapéuticas sin alivio significativo del dolor. Los pacientes fueron entrevistados y se les solicitó evaluar el dolor y las actividades de la vida diaria, comparando antes y después del tratamiento con opiáceo intratecal. La infusión de opiáceo intratecal resulto en alivio del dolor. Sim embargo, la mejoría de la funcionalidad no fue observada con la misma magnitud.

TÉRMINOS CLAVES: dolor, dolor crónico, opiáceo, alivio del dolor, actividades de la vida diaria, dolor neuropático, dolor miofacial

\section{REFERÊNCIAS BIBLIOGRÁFICAS}

\section{AGENCY FOR HEALTH CARE POLICY AND} RESEARCH. Acute pain management: operative or medical procedures and trauma. Rockville, 1992.

02. FINLAYSON, R.E; MARUTA, T.; MORSE, R.M.; MARTIN, M.A. Substance dependence and chronic pain: experience with treatment and follow-up results. Pain, v. 26, p. 175-80, 1986.

03. FOLEY, K.M. Opioids. Neurol. Clin., v. 11, n. 3, p. 503-22, 1993.

04. GILDENBERG, P.L.; DEVAUL, R.A. O paciente de dor crônica: avaliação e tratamento. Rio de Janeiro: Colina, 1987.

05. HANKS, G.W.; JUSTINS, D.M. Cancer pain: management. Lancet, v. 339, p. 1031-5, 1992.

06. JACOX, A. et al. Management of cancer pain. Rockville; U.S.: Departament of Health and Human Services, 1994. p. 52-3 (AHCPR Publication, n. 94-052).

07. MARUTA, T.; SWANSON, D.W.; FINLAYSON, R.E. Drug abuse and dependency in patients with chronic pain. Mayo Clin Proc., v. 54, p. 241-4, 1979.

08. MOULIN, D.E.; IEZZI, A.; AMIREH, R.; SHARPE, W.K.J.; BOYD, D.; MERSKEY, H. Randomised trial of oral morphine for chronic non-cancer pain. Lancet, v. 347, p. 143-7, Jan. 1996.

09. ORGANIZACION MUNDIAL DE LA SALUD. Alivio del dolor en el cancer. Ginebra, 1987.

10. PORTENOY, R.K. Chronic opioid therapy in nonmalignant pain. J.Pain Symptom Management, v. 5, n. 1, p. 46-62, 1990. Suppl.

11. PORTENOY, R.K.; FOLEY, K.M. Chronic use of opioid analgesics in non-malignant pain: report of 38 cases. Pain, v. 25, p. 171-86, 1986.

12. SAVAGE, S.R. Addiction in the treatment of pain: significance, recognition and management. J.Pain Symptom Management, v. 8, n. 5, p. 26578, 1993.
13. SAVAGE, S.R. Long-term opioid therapy: assesssment of consequences and risks. J.Pain Symptom Management, v. 11, n. 5, p. 274-86, 1996.

14. SCHOFFERMAN, J. Long-term use of opioid analgesics for the treatment od chronic pain nonmalignant origin. J.Pain Symptom Management, v. 8, n. 5, p. 279-88, 1993.

15. SCHUG, S.A.; LARGE, R.G. Opioids for chronic noncancer pain. Pain Clin. Updates, v. 3, n. 3, Nov. 1995. 4p.

16. SCHULZECK, S.; GLEIM, M.; MAIER, C. Factors contributing to the results of long-term treatment with oral morphine tablets in patients with chronic non-malignant pain. Anaesthesist, v. 42, p. 545$56,1993$.

17. SEES, K.L.; CLARK, H.W. Opioid use in the treatment of chronic pain: assessment of addiction. J.Pain Symptom Management, v. 8, n. 5, p. 257-64, 1993.

18. STEIN, C. Morphine: a local analgesic. Pain Clin. Updates, v. 3, n. 1, March 1995. 4p.

19. TAYLOR, C.B.; ZLUTNICK, S.I.; CORLEY, M.J.; FLORA, J. The effects od detoxication, relaxation and brief supportive therapy on chronic pain. Pain, v. 8, p. 319-29, 1980.

20. TENNANT, F.S.; UELMEN, J.D. Narcotic maintenance for chronic pain. Medical and legal guidelines. Posgraduate Medicine, v. 73, n. 1, p. 81-94, 1983.

21. TURK, D.C.; BRODY, M.C.; OKIFUJI, E.A. Physicians'attitudes and practice regarding the long-term prescribing of opioids for non-cancer pain. Pain, v. 59, p. 201-8, 1994.

22. ZENS, M.; STRUMPF, M.; TRYBA, M. Long-term oral opioid therapy in patients with chronic nonmalignant pain. J.Pain Symptom Management, v. 7, n. 2, p. 69-77, 1992. 